\title{
Self-validating Contact Thermometry Sensors for Higher Temperatures
}

\author{
Frank Edler $^{1}$, Peter Seefeld ${ }^{2}$, Guillaume Failleau ${ }^{3}$ \\ ${ }^{1}$ Physikalisch-Technische Bundesanstalt, Abbestraße 2-12, D-10587 Berlin, Germany, \\ frank.edler@ptb.de \\ ${ }^{2}$ E+H Endress + Hauser Wetzer GmbH Co KG, Obere Wank 1, D-87484 Nesselwang, Germany, \\ ${ }^{3}$ Laboratoire Commun de Métrologie LNE-Cnam, 61 rue du Landy, F-93210 Saint-Denis La Plaine
}

\begin{abstract}
:
This paper is focused on self-validation and in-situ validation methods of thermometry between $1000{ }^{\circ} \mathrm{C}$ and about $1800{ }^{\circ} \mathrm{C}$. An operative approach of reliable self-validating contact thermometry sensors and the implementation of traceable measurement methods for the measurement of temperatures up to about $1800{ }^{\circ} \mathrm{C}$ in oxidizing atmospheres will be presented. Miniature fixed points filled with high purity $\mathrm{Au}\left(1064.18^{\circ} \mathrm{C}\right), \mathrm{Pd}\left(1553.4^{\circ} \mathrm{C}\right)$ and $\mathrm{Pt}\left(1769{ }^{\circ} \mathrm{C}\right)$ have been constructed and assembled with type $B$ thermocouples to be used as traceable references in oxidizing atmospheres. The use of electrical noise thermometry as an additional method for realization of the self-validation principle was applied. Combined thermocouple-noise temperature sensors have been constructed to allow long-term determination of thermocouple drift. Electrical noise thermometry is a primary method to measure temperatures without drift effects even over long periods of time. An uncertainty of noise temperatures measured of $0.1 \%$ has been achieved which is sufficient to determine and correct for thermocouple drifts in industrial use.
\end{abstract}

Key words: Thermocouple, Miniature fixed point, Noise thermometry, Self-validation

\section{Introduction}

Self-validated measurements of temperatures above $1000^{\circ} \mathrm{C}$ are both difficult and vital for the feasibility and the success of industrial processes e.g. for the manufacture of silicon, carbides, carbon/carbon composites, iron, steel, glass and ceramics. Many of these sectors of industry require improved process efficiency/control, also because of growing environmental concerns (emissions/"zero waste") and ex-EU competition. One of the keys to making advances to these drivers is improving process control by improved high temperature measurement. [1]

Improvements in sensing methods, especially in-situ validation, may bring about a step change improvement in the practice of thermometry and hence in industrial process control by using lower uncertainties of the installed temperature sensors.

The self-validation concepts presented in this paper are based on the one hand on the use of miniature fixed points with defined and stable melting temperatures of pure metals which are combined directly with commonly used thermocouples to detect their drift effects, similar as described in $[2,3]$ for lower temperatures. On the other hand, the second self-validation concept is based on the simultaneous use of a further (primary) method to measure temperatures without drift effects to validate for instance the thermoelectric stability of thermocouples as parts of a combined thermocouple-noise temperature sensor. Electrical noise thermometry was successful used for metrological applications [4], but had demonstrated its ability also for industrial applications [5].

\section{Cell design of the miniature fixed points}

Four miniature fixed-point crucibles have been constructed at PTB to be used as reference artifacts for type $B$ thermocouples which are usable to temperatures of about $1800{ }^{\circ} \mathrm{C}$ in oxidizing atmospheres. Two of the cells were filled with palladium and the other two were filled with platinum. The miniature fixed-point crucibles are made of high purity $(99.7 \%)$ Alumina $\left(\mathrm{Al}_{2} \mathrm{O}_{3}\right)$ and were annealed at about $1300{ }^{\circ} \mathrm{C}$ for several hours in air before filling. Miniature fixed-point cells of the two different designs A and B constructed at PTB are shown in Figures 1 and 2. 


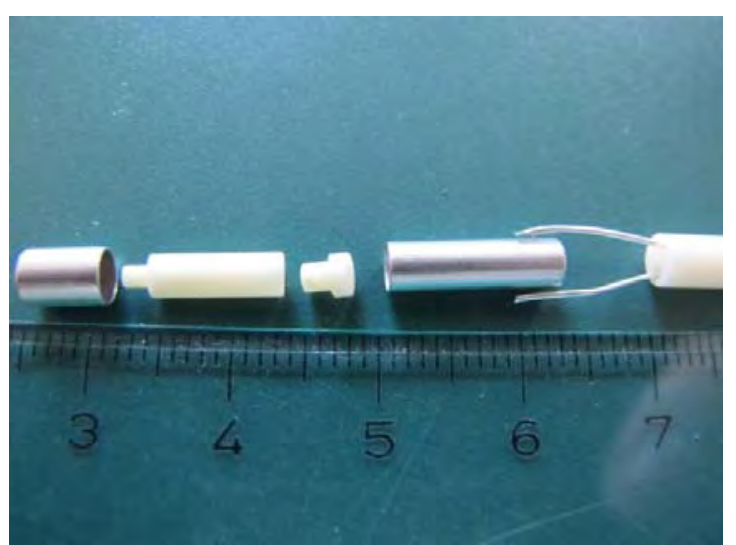

Fig. 1. Miniature fixed point of design A.

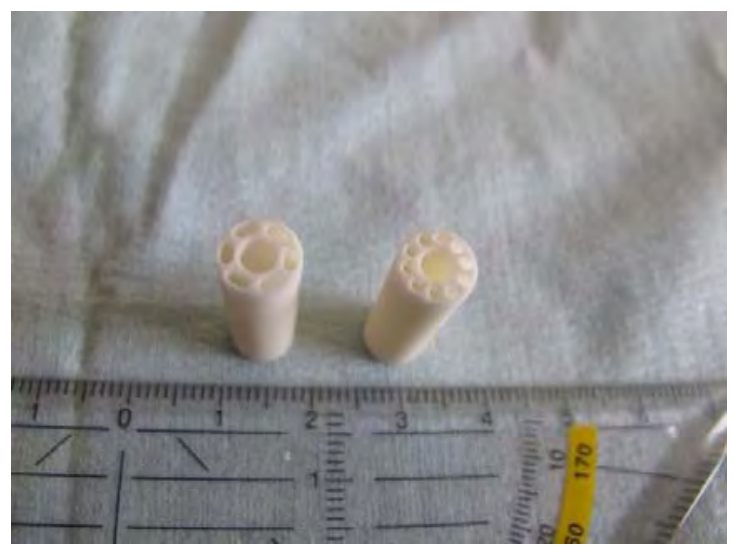

Fig. 2. Miniature fixed points of design B (left crucible filled with $P t$, right crucible filled with $P d$ ).

The miniature fixed points of the design $A$ have been constructed similar to the fixed-point cells described in [6]. The ceramic crucible containing the fixed-point metal was inserted into an ancillary cartridge made of a PtRh alloy which was welded between the two thermoelements of the type $\mathrm{B}$ thermocouples. The miniature fixed point $\mathrm{Pd}-01-12$ contains $0.23 \mathrm{~g}$ of high purity $(99.99 \%)$ palladium, the miniature fixed point Pt-01-12 contains $0.53 \mathrm{~g}$ of high purity $(99.997 \%)$ platinum. The outer diameter of the ceramic crucibles amounts to a value of $3 \mathrm{~mm}$. Their length is about $10 \mathrm{~mm}$. These small dimensions allowed a simple integration of the fixed-point cells in a ceramic protection tube of $7 \mathrm{~mm}$ outer and $5 \mathrm{~mm}$ inner diameter typically used for standard thermocouples.

The miniature fixed points of design $B$ have an outer diameter of $7.2 \mathrm{~mm}$ and a length of 22 $\mathrm{mm}$. The insulation tube (3 $\mathrm{mm}$ in diameter) of the thermocouple with the measuring junction was inserted close-fitting into the central bore (3.2 $\mathrm{mm}$ in diameter) of the fixed-point cell. The outer bores (or slots) of $1.1 \mathrm{~mm}$ in diameter contain the fixed-point material in form of wires: Pd-02-12 contains a total mass of $1.4 \mathrm{~g}$ of high purity (99.95\%) palladium and Pt-B-02-12 contains a total mass of about $2 \mathrm{~g}$ of high purity (99.99\%) platinum.

A self-validating device proposed by LNE-Cnam is based on the pulled-wire method. A fixedpoint cell is integrated in a type $B$ thermocouple for enabling an in-situ monitoring of the sensor drift during a process. In the four-hole insulation tube of the thermocouple two holes are used for the thermoelements and the other two are filled with the fixed-point material. The principle construction is depicted in the Figure 3: the pure fixed-point material (gold or palladium in this study) shaped as wires with $0.5 \mathrm{~mm}$ in diameter are pulled in the two empty bores of the insulation tube. It is machined in order to place the thermocouple hot junction at the middle of the pure metal wires length. A ceramic adhesive (pure alumina based) enable to encapsulate the pure metal on the capillary extremity.

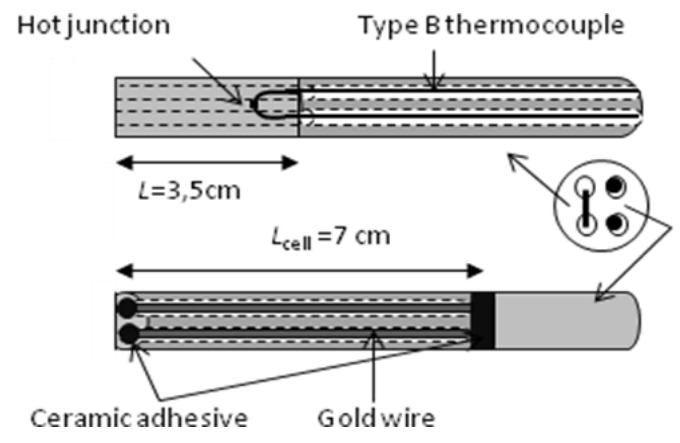

Fig. 3. Schematic description of the pulled-wire integrated fixed-point design

\section{Melting behavior of the integrated miniature fixed points}

A typical melting curve of palladium $\left(T_{S}=\right.$ $1553.4{ }^{\circ} \mathrm{C}$ ) by using the type $\mathrm{B}$ thermocouple SV-B-Pd-01-12 with the integrated fixed-point crucible Pd-01-12 (design A) against the emf of the furnace control thermocouple is shown in Figure 4. The melting curve shows a constant increase of the emf before the melting process starts. During the melt the slope is decreased. Both parts of the melting curve can be approximated by regression lines, respectively. The intersection point of the two straight regression lines corresponds to the emf of the melting point. The sudden reduced slope indicates the beginning of the melt; the rapid increase of the slope marks the end of the melt.

A more clear detection of the start of the melt is achieved by calculating the differential emfs $(\Delta \mathrm{emf})$ between the emfs of the test thermocouple and the control thermocouple ( $y$ axis) against the emf of the test thermocouple (x-axis) as shown in Figure 5. Here, the change 
of the slope is more significant and the regression lines can be fitted unambiguous.

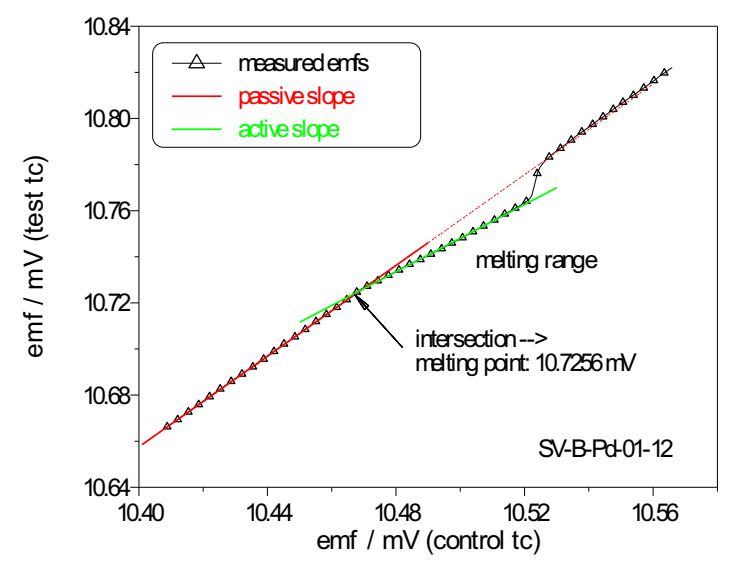

Fig. 4. Typical melting curve of palladium

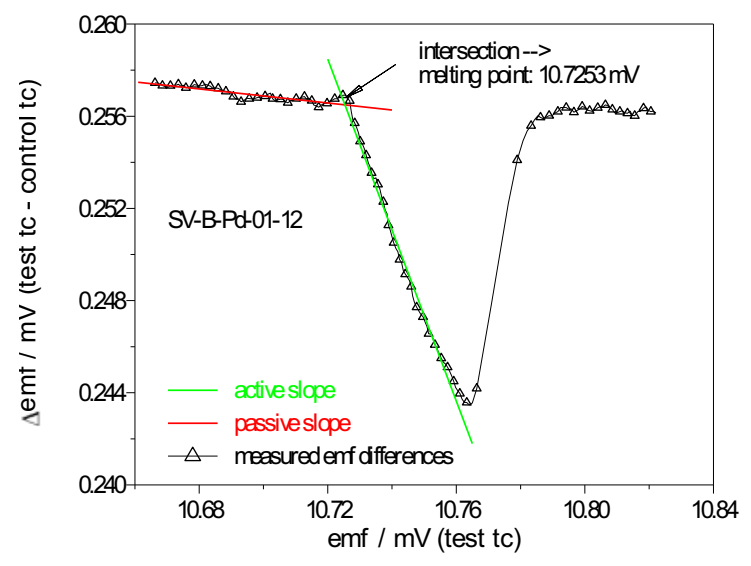

Fig. 5. Differential melting curve of palladium

A special feature of the melt by using the integrated fixed-point cells of design $A$ was the dependency of the melting temperature on the heating rate, as visible in Figure 6.

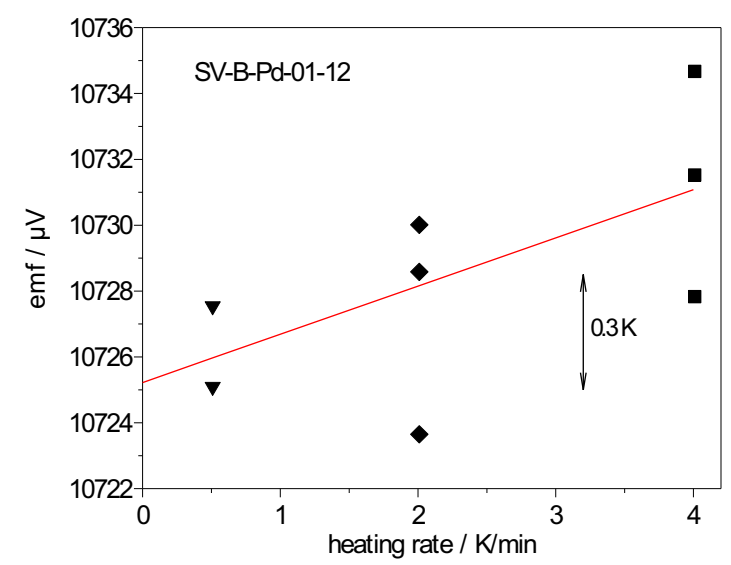

Fig. 6. Dependency of the melting temperatures on the heating rate by using the miniature fixed point $P d-01-12$ of design $A$

This demonstrates a limit of this method. Since the hot junction it is not surrounded by the fixed-point material, the measurement is sensitive to the heat flux from the furnace. Therefore, an extrapolation of the results to adiabatic conditions (heating rate of $0 \mathrm{~K} / \mathrm{min}$ ) seems to be necessary. Otherwise, this effect could be integrated into the uncertainty budget which would increase the combined uncertainty slightly.

Similar effects were obtained at LNE-Cnam by using the type B thermocouple (LNE/PW01/Au) which has been assembled for operating up to the melting point of gold $\left(1064.62{ }^{\circ} \mathrm{C}\right)$. The mass of gold amounts to a total of $0.8 \mathrm{~g}$. The melting temperatures and curves observed depend on the offset temperature of the furnace $(3 \mathrm{~K}, 6 \mathrm{~K}$, and $12 \mathrm{~K}$ ) to realize this phase transition as shown in Figure 7.

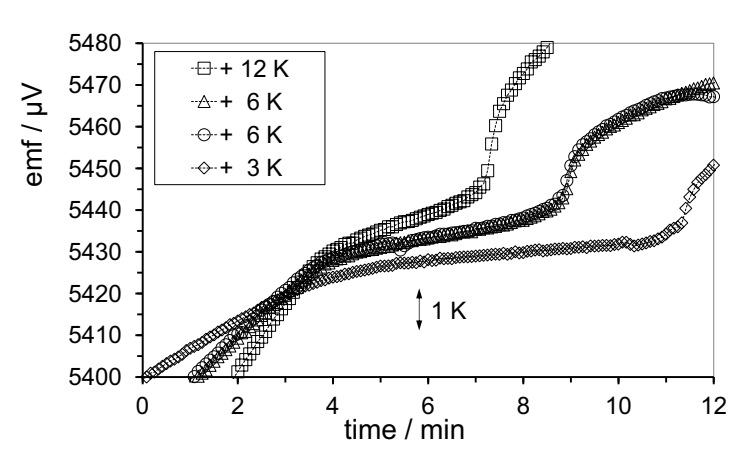

Fig. 7. Dependency of the melting temperatures on the offset temperatures of the furnace by using the LNE-Cnam design of a Au miniature fixed point

The melting temperatures of platinum ( $T_{\mathrm{S}}=$ $1769{ }^{\circ} \mathrm{C}$ ) by using the type $\mathrm{B}$ thermocouple (SV-B-Pt-02-12) with the integrated fixed-point crucible Pt-02-12 of design B were almost independent of environmental conditions. Here, the fixed-point material surrounds the measuring junction at least partly. Two melting curves with different heating rates (by a factor of three) but resulting in about the same melting temperatures are presented in Figure 8 for illustration.

The melting temperatures of pure metals of integrated miniature fixed-point cells can be used as reference temperatures for selfvalidation of thermocouples. However, some specific features must be considered: the influence on the melting temperature of environmental conditions (heating rate, offset temperatures of furnaces) and the assignment of the melting temperature to the melting curve whose shapes are often no flat plateaus. Keeping this in mind, drift effects of thermocouples which exceed the uncertainty of the determination of melting temperatures by 
using the miniature fixed points can be validated in-situ and corrected for further use.

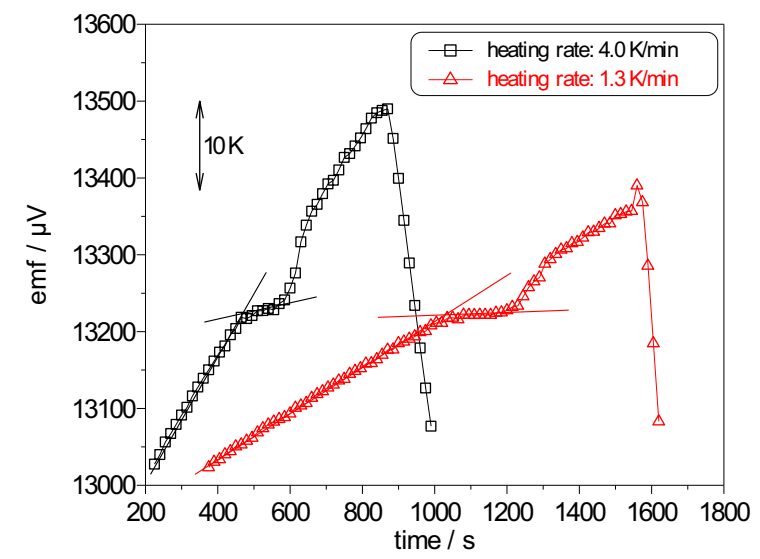

Fig. 8. Melting curves of platinum by using the miniature fixed point Pt-02-12 of design $B$

\section{Electrical noise thermometry}

Noise thermometry uses the random thermal movement of the electrons in the conduction band of a metal to measure thermodynamic temperatures. It is based quantitatively on the Nyquist formula (1) and is independent of temperature depending material properties:

$$
\overline{U^{2}}=4 k T R \Delta f
$$

where $\overline{U^{2}}$ is the mean square noise voltage, $k$ the Boltzmann-constant, $T$ the thermodynamic temperature, $R$ the ohmic resistance and $\Delta f$ the noise bandwidth over which the noise voltage is measured. Equation (1) is seldom used directly because parasitic noise sources (amplifiers, leads) are included, because of difficulties in measuring the equivalent noise bandwidth, and because of the necessity of the calibration of the gain of the measuring system [4]. Therefore, a comparison method by using reference resistors $R_{\mathrm{R}}$ and a correlation technique by using two identical channels of parallel amplifiers are applied [7]. The noise temperature $T_{\mathrm{S}}$ is determined by comparing the mean square noise voltage of the reference resistor $R_{R}$ at a known reference temperature $T_{\mathrm{R}}$ with the mean square noise voltage of the measuring resistor $R_{\mathrm{S}}$ within the same bandwidth $\Delta f$ according to equation (2):

$$
T_{\mathrm{S}}=\overline{U_{S}^{2}} \overline{U_{R}^{2}} \cdot R_{\mathrm{R}} / R_{\mathrm{S}} \cdot T_{\mathrm{R}}
$$

Due to the stochastic nature of the thermal noise, the accuracy of the noise temperature measurement depends on the measuring time $T$ and the bandwidth $\Delta f$. The relative uncertainty of the noise temperature also takes into account the additional amplifier chain's internal noises of the two channels and is given by equation (3) [7]:

$$
\Delta T / T=\left[2 \cdot\left(2+\mathrm{B} / \mathrm{A}+\mathrm{C} / \mathrm{A}+\mathrm{BC} / \mathrm{A}^{2}\right) /(\Delta f \cdot T)\right]^{1 / 2}
$$

where $A$ is the power spectral density of the measuring or the reference resistor $\left(R_{\mathrm{S}} \cdot T_{\mathrm{S}}\right.$ or $\left.R_{\mathrm{R}} \cdot T_{\mathrm{R}}\right)$ and $\mathrm{B}$ and $\mathrm{C}$ are the power spectral densities of the amplifier chain's internal noise of the two channels. The ratios B/A and C/A were in the order of 1 to 1.2 for all noise temperature measurements performed in this study.

One combined thermocouple-noise temperature sensor (RT-B1) consisting of two type B thermocouples and a second combined thermocouple-noise temperature sensor (RTS1) consisting of two type $S$ thermocouples have been constructed as described in [4] to investigate and to validate the thermal stability of the thermocouples used. They can be used in air up to temperatures of $1800{ }^{\circ} \mathrm{C}$ and $1500{ }^{\circ} \mathrm{C}$, respectively. Measurements were performed at the freezing points of copper $\left(1084.62{ }^{\circ} \mathrm{C}\right)$ and silver $\left(961.78{ }^{\circ} \mathrm{C}\right)$ by using two different, independent Noise-ThermometerElectronics (NTE) of PTB and $\mathrm{E}+\mathrm{H}[4,5]$ to test the accuracy of the combined thermocouplenoise sensors. The mean noise temperatures measured agree to the well known fixed-point temperatures (ITS-90) within about $0.4 \mathrm{~K}$ at the freezing point of copper and within $0.2 \mathrm{~K}$ at the freezing point of silver. These results demonstrate the suitability for the intended use of the combined thermocouple-noise temperature sensors based on type $B$ and type $S$ thermocouples as a suitable tool to detect and correct for drifts in the thermocouples within the relative target uncertainty of $0.1 \%$. The noise temperatures measured at the two freezing points are presented in Figures 9 and 10. The error bars corresponds to the statistical uncertainty $(k=2)$, of the noise temperature calculated by using eq. (3).

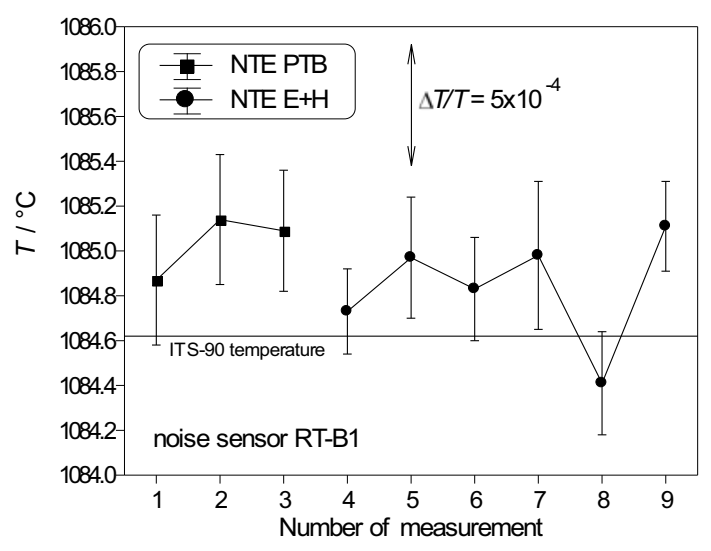

Fig. 9. Noise temperatures at the freezing point of copper by using sensor RT-B1 


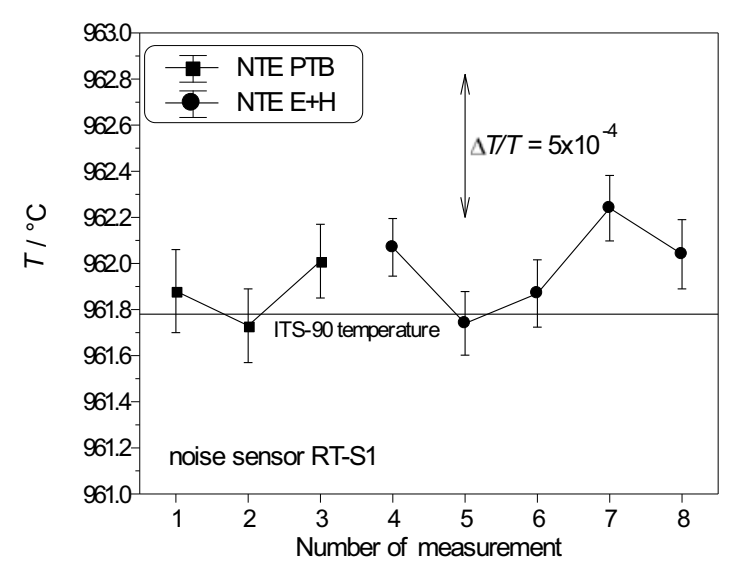

Fig. 10. Noise temperatures at the freezing point of silver by using sensor RT-S1

The two type $B$ thermocouples of the combined thermocouple-noise temperature sensor RT-B1 were calibrated at the freezing point of copper; the type $S$ thermocouples of the sensor RT-S1 were calibrated at the freezing point of silver. In this way additional reference values were established to confirm possible changes of the thermoelectric stability of the thermocouples which should be detected by the noise temperature sensor.

A first thermal treatment of the combined thermocouple-noise sensor RT-B1 at $1450{ }^{\circ} \mathrm{C}$ for about 80 hours under laboratory conditions caused an increase of the emfs at the freezing point of copper of the two type $B$ thermocouples by about $3.3 \mu \mathrm{V}(0.35 \mathrm{~K})$ and $4.7 \mu \mathrm{V}(0.5 \mathrm{~K})$, respectively. This is less than $0.1 \%$ and therefore in the order of the measurement uncertainty of the noise temperature, i.e. below the limit of detection.

Beside the statistical uncertainty of noise temperature measurements other influences have to be considered. The noise temperature is calculated according to eq. (1). Therefore, the uncertainty of the electrical measurement of the parameters $\left(R_{\mathrm{S}}, R_{\mathrm{R}}\right.$, and $\left.T_{\mathrm{R}}\right)$ has to be taken into account. The values of the mean square noise voltages are influenced by electromagnetic interference (EMI) which can be superimposed on the useful noise signal. Interferences in the frequency range are visible in the temperature spectra and could be removed subsequently by filtering (rejecting disturbed frequency ranges). Remaining interference was less than the limits of detection which are in the order of $1 \cdot 10^{-4}$. Transmission errors of the noise voltages caused by a possible mismatch of the impedance, $Z_{W}$, of the transmission lines (thermoelements) and the measuring resistance, $R_{\mathrm{S}}$, also could be estimated from the recorded averaged temperature spectra within about $1.10^{-4}$, because these errors depends on frequency. Non-linearity effects of electronic components (amplifiers) can be reduced significantly $\left(<5 \cdot 10^{-5}\right)$ by choosing the value of the reference resistors in this way that its mean square noise voltage corresponds to about the same value like the mean square noise voltage of the measurement resistor, i.e. $\overline{U_{S}^{2}} \overline{U_{R}^{2}}=1$. A more detailed description and estimation of uncertainty contributions of noise temperature measurements can be found in [8].

The good performance of the combined thermocouple-noise temperature sensor RT-B1 is shown in Figure 11. The seven hour lasting measurement in the temperature range between $1438{ }^{\circ} \mathrm{C}$ and $1428{ }^{\circ} \mathrm{C}$ demonstrates the very good agreement between the two thermocouple temperatures and the noise temperature. This confirms again the suitability of this self-validating concept to detect possible drifts of thermocouples in the order of about $0.1 \%$. The statistic uncertainty $(k=2)$ of one measuring point corresponds to about $\pm 1.2 \mathrm{~K}$ with an effective measuring time $T$ of 330 seconds within a frequency interval of about $270 \mathrm{kHz}$.

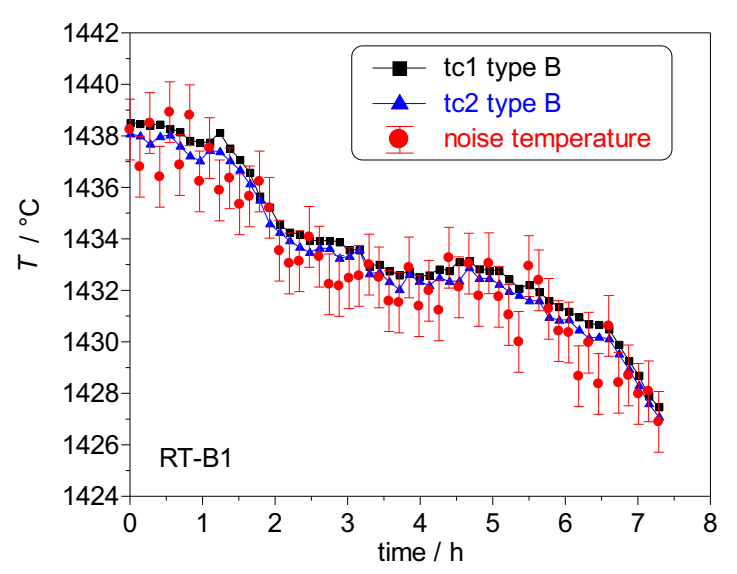

Fig. 11. Temperature course by using the combined thermocouple-noise temperature sensor RT-B1 consisting of the two type $B$ thermocouples tc 1 and tc2

\section{Conclusion}

The melting curves measured by using the thermocouples with integrated miniature fixed points allow a unambiguous assignment of the corresponding melting temperatures provided that agreed methods for analysis of the curves are used, for instance the application of regression lines. The measurement uncertainty of the melting temperatures increases with increasing temperatures and is in the order of (1-2) $\mathrm{K}$ at the melting points of palladium and platinum. Therefore, drift effects of 
thermocouples less than about $(1-2) \cdot 10^{-3}$ won't be detectable.

Similar detection limits are attainable by using noise thermometry as an independent method if a sufficient measurement time $t$ is applicable.

In principle, both methods appear promising as self validating concepts to detect drift effects insitu, therefore their practical suitability should be confirmed by further investigations.

\section{Acknowledgement}

This work was founded by the European Metrology Research Programme (EMRP) and national metrology research programmes.

\section{References}

[1] G. Machin, K. Anhalt, F. Edler, J. Pearce, M. Sadli, R. Strnad, and E. Vuelban, 2012 HiTeMS: a project to solve high temperature measurement problems in industry, 9th Int. Temperature Symp. (ITS9) (Los Angeles, 19-23 March 2012)

[2] S. Augustin and F. Bernhard et al, Industrially applicable miniature fixed point thermocouples Proceeding of TEMPMEKO 2001, 8th International Symposium on Temperature and
Thermal Measurements in Industry and Science, 2001, pp.3-8.

[3] H. Lehmann, Fixed-point thermocouples in power plants: long term operational experiences, Int. J. Thermophys., 31, 1599-1607 (2010)

[4] F. Edler, M. Kühne and E. Tegeler, Noise temperature measurements for the determination of the thermodynamic temperature of the melting point of palladium, Metrologia 41, (2004) 47-55

[5] P. Seefeld, S. Damith, M. Konrad, M. Korn, A. Zotz, Prozessgeeignete Rauschtemperaturmessungen, 15th ITG/GMA conference, Proceedings of Sensoren und Messsysteme 2010, pp. 211-216

[6] M. Tischler, M.J. Koremblit, Miniature Thermometric Fixed Points for Thermocouple Calibrations, Temperature: Its Measurement and Control in Science and Industry (Ed. Schooley), 5, AIP, New York 1982, pp. 383-390

[7] H. Brixy, Noise Thermometry, Sensors, Vol.4, Thermal Sensors, ed. by T. Ricolfi and J. Scholz, VCH Verlagsgesellschaft, 1990, pp. 225-249

[8] F. Edler, E. Tegeler, E Zimmermann, Thermodynamic Temperature of the Freezing Point of Copper Measured by Noise Thermometry, Temperature: Its Measurement and Control in Science and Industry (Ed. D.C. Ripple), AIP conference proceedings 684, pp 1318, (2003); doi: 10.1063/1.1627095 